\title{
Decompressive craniotomy for the treatment of malignant infarction of the middle cerebral artery: mortality and outcome
}

\author{
Craniotomia descompressiva para tratamento do infarto maligno da artéria cerebral \\ media: mortalidade e desfecho
}

Gianise Toboliski Bongiorni', Marjeane Cristina Jaques Hockmuller', Cristini Klein², Ápio Cláudio Martins Antunes ${ }^{3}$

\begin{abstract}
Objective: To assess, by Rankin scale, the functional disability of patients who had a malignant middle cerebral artery (MCA) ischemic stroke, who underwent decompressive craniotomy (DC) within the first 30 days. Methods: A cross-sectional study in a University hospital. Between June 2007 and December 2014, we retrospectively analyzed the records of all patients submitted to DC due to a malignant MCA infarction. The mortality rate was defined during the hospitalization period. The modified outcome Rankin score (mRS) was measured 30 days after the procedure, for stratification of the quality of life. Results: The DC mortality rate was $30 \%$ (95\% Cl 14.5 to 51.9 ) for the 20 patients reported. The mRS 30 days postoperatively was $\geq 4$ [3.3 to 6] for all patients thereafter. Conclusion: DC is to be considered a real alternative for the treatment of patients with a malignant ischemic MCA infarction.
\end{abstract}

Keywords: craniotomy; cerebral infarction; intracranial hypertension.

RESUMO

Objetivo: Avaliar a capacidade funcional de pacientes com acidente vascular cerebral isquêmico no território da artéria cerebral média (ACM) submetidos à craniotomia descompressiva (CD) no período de 30 dias pela escala de Rankin. Métodos: Estudo transversal em um hospital universitário. Entre junho de 2007 e dezembro de 2014, analisados retrospectivamente os registros de todos os pacientes submetidos a CD devido a enfarte maligno na ACM. A taxa de mortalidade foi definida durante o período de internação. O resultado da estratificação da qualidade de vida foi através da escala Rankin modificado (mRS) mensurado em 30 dias após o procedimento. Resultados: A taxa de mortalidade CD foi de 30\% (IC 95\% 14,5-51,9) para os 20 pacientes relatados. A mRS 30 dias de pós-operatório foi $=>4$ [3,3-6] para todos os pacientes. Conclusão: $C D$ deve ser considerada uma alternativa real para o tratamento de pacientes com enfarte isquêmico no território da ACM.

Palavras-chave: craniotomia; infarto cerebral; hipertensão intracraniana.

A decompressive craniotomy (DC) has been a therapeutic option for an infarction of the middle cerebral artery (MCA) region. The goal of the surgical treatment is the reduction of the intracranial hypertension. The procedure is based on a fronto-parieto-temporal craniotomy, ipsilateral to the lesion, followed by plastic reconstruction of the dura mater, allowing immediate decompression of the brain $1,2,3,4,5$.

Some complications, such as infection of the surgical area, temporal muscle hematoma with cerebral compression, intracerebral hemorrhage, or worsening of the neurological condition may follow $\mathrm{DC}^{2,3,4}$. In order to clearly define the indication for the surgical treatment and to decrease complications derived from DC in a university hospital in southern Brazil, a specific protocol for DC in the treatment of intracranial hypertension derived from ischemia of the MCA region has been implemented since 2007, in conjunction with the neurology department. Even though studies have demonstrated the efficacy of DC in patients presenting with malignant MCA infarction ${ }^{56,678,8,10}$, some resistance to its application still exists, particularly regarding the functionality of the patients who survive the acute event.

The goals of the present study were to assess the mortality rates from the use of DC in the treatment of patients

\footnotetext{
${ }^{1}$ Universidade Federal do Rio Grande do Sul, Programa de Pós-Graduação em Ciências Médicas - Ciência Cirúrgica, Porto Alegre RS, Brasil;

${ }^{2}$ Universidade Federal do Rio Grande do Sul, Programa de Pós-Graduação em Ciências Médicas, Porto Alegre RS, Brasil;

${ }^{3}$ Universidade Federal do Rio Grande do Sul, Hospital de Clínicas de Porto Alegre, Serviço de Neurocirurgia, Porto Alegre RS, Brasil.

Correspondence: Gianise Toboliski Bongiorni; Hospital de Clínicas de Porto Alegre;

Rua Ramiro Barcelos, 2350; 90035-007 Porto Alegre RS, Brasil; E-mail: gianise1@hotmail.com

Conflict of interest: There is no conflict of interest to declare.

Received 30 November 2016; Accepted 14 February 2017.
} 
with intracranial hypertension due to an ischemic infarction of the MCA region. A secondary objective was to assess the quality of life of the patients discharged from hospital after the procedure, by means of the modified Rankin scale (mRS) score, 30 days after discharge from the hospital.

\section{METHODS}

A retrospective cohort study was performed in a single center setting, in a public teaching hospital. All patients subjected to DC for the treatment of intracranial hypertension in the MCA region, between June 2007 and December 2014, were included. Data were extracted from electronic records, including general patient characteristics, clinical and surgical variables: time elapsed from the ictus to admission in the Hospital de Clínicas de Porto Alegre emergency unit, National Institutes of Health Stroke Scale (NIHSS) score upon admission, time elapsed between the first neurosurgery evaluation and the therapeutic decision to proceed with DC, interval in hours between the primary event and the surgery, clinical worsening after the first neurosurgery evaluation, number of CT scans necessary for the surgical decision, time interval between the last $\mathrm{CT}$ and the $\mathrm{DC}, \mathrm{mRS}$ score at the time of hospital discharge and after 30 days, the outcome after DC (discharge/death); and the in-hospital time interval.

The sample consisted of 20 patients, corresponding to the total number of patients submitted to DC in the referred period. For the surgical indication, the institutional protocol is detailed in Table 1.

\section{Surgical technique}

The following were the stages of the surgery. Patient was positioned in dorsal decubitus. Head pin-fixed, elevated $60^{\circ}$ and rotated approximately $90^{\circ}$, to make it parallel to the floor. Elevation of the ipsilateral shoulder. Upper and lower limbs were stretched out along the body. Surgical asepsis was done with chlorhexidine, followed by alcohol chlorhexidine. Demarcation of the surgical incision with a dermographic pen, in a curved line, extending from the frontal region (about 1 to $2 \mathrm{~cm}$ away from the midline), involving the parietal region and ending at the base of the ipsilateral temporal bone. Placement of surgical towels. Skin incision, following the previous delimited marking. Hemostasis and divulsion of the planes (some authors advocate the use of a single plane), with preservation of the ipsilateral temporal artery, as well as the frontal branch of the facial nerve. Identification and incision the temporal muscle fascia: opening in a single plane or not. Detachment of the temporal muscle from the bone plate and traction. Bone wax hemostasis. Craniotomy, preferably with a craniotome, with previous demarcation of the main points, such as the inion, with preservation of the frontal sinuses. Detachment of the bone plate. Hemostasis of the bone edges with bone wax. Dural opening in arc, with
Table 1. Institutional decompressive craniotomy protocol.

\section{Protocol inclusion criteria}

National Institutes of Health Stroke Scale (NIHSS) > 15, clinical evidence of MCA extensive infarction (complete hemiparesis contralateral to the lesion, homonymous hemianopsia contralateral to the lesion, tendency for oculocephalic deviation towards the lesion, language alteration, heminegligence);

Age $\leq 60$ years (in patients older than 60 years, the decision must be individualized);

Tomographic evidence of early hypodensity involving corticosubcortical topography $>50 \%$ of the MCA territory.

\section{Protocol exclusion criteria}

Other previous incapacitating diseases (skeletal muscle, neurologic or clinical);

Signs of severe neurological deterioration at the time of surgical indication (unilateral or bilateral pupil dilatation, with mydriasis or absence of photo-reaction, and/or signs of pathological extension or flexion);

Severe clinical complications;

Terminal illness;

$m R S \geq 3$;

Clotting disorders;

Unavailability of the intensive care unit (ICU) beds;

Hemodynamic instability at the time of surgical indication.

\section{Patient initial follow-up}

Second CT between 6-12 hours after patient arrival, or less, in the case of neurological deterioration - early detection of the malignant evolution;

After the second CT: new CT every six to eight hours.

Indication for decompressive surgery

Any neurological deterioration in relation to the admission or history of neurological deterioration since the beginning of the symptoms;

Any tomographic worsening (extension of the infarction or worsening of the space-occupying effect).

Levels of evidence of the recommendations

Early DC is recommended for the treatment of MCA space occupying cerebral infarction in patients up to 60 years of age, before the manifestation of clinical or radiological signs of cerebral herniation, within the time span of 48 hours (evidence level 1, recommendation grade A - Systematic Review (with homogeneity) of controlled and randomized clinical trials).

The decision of DC for patients older than 60 years of age or after 48 hours must be individualized. (evidence level 4 , recommendation grade $\mathrm{C}$ - case reports (including cohort or case control of lesser quality).

The independent variables studied were: age, sex, marital status, time elapsed between ictus and surgery, first CT and surgery and the score of the National Institutes of Health Stroke Scale (NIHSS) upon admission.

The mRS evaluates the functionality of the individual after a CVA (either ischemic or hemorrhagic) and it became an important clinical tool capable of estimating the prognosis and the outcome. It comprises seven items, with a score of zero being considered without symptoms, and a score of 6 being death (Addendum 1).

The primary regarded outcomes were death and hospital discharge: surgical deaths were considered up to 30 days after the DC operation. For every patient, the mRS scores were measured: those that died scored 6; patients discharged from hospital within 30 days had their score measured at that time. 
the base towards the parietal lobe, or in a cruciform shape. Hemostasis and dural anchoring to the bone edges. Brain irrigation with warm saline solution. Dural opening in a cruciate fashion, followed by duraplasty, preferably with aponeurotic galea previously prepared or fascia lata, taken from the lateral vastus muscle, with continuous stitches and sealed with biological glue. Placement of a closed draining system. Subcutaneous tissues and skin closing. Head dressing with surgical gauze, bandage and adhesive tape, with a label: "WITHOUT BONE FLAP". Straight incision in the upper quadrant of the ipsilateral hemiabdomen. Hemostasis and identification of the subcutaneous tissue. Identification, hemostasis and detachment of the fat tissue. Identification of the muscular fascia. Insertion of the bone flap under the muscular fascia. Careful review of the hemostasis. Suture by planes. Simple abdominal dressing.

\section{Statistical analysis}

The data analysis was done using the Statistical Package for Social Sciences (SPSS) 18.0. The categorical variables were expressed as absolute value and percentage; the continuous variables as average \pm standard deviation or median and interquartile ranges, according to data distribution. The Kaplan-Meier curve was created to report the in-hospital mortality.

\section{RESULTS}

In the period of the study, a total of 20 patients were subjected to DC for the treatment of intracranial hypertension due to malignant MCA ischemia. The socio-demographic characteristics of the patients included are shown in Table 2.

The time elapsed between the ictus and the admission to the emergency unit of the Hospital de Clínicas de Porto Alegre was an average of $13.2 \pm 1.4$ hours: in two patients, it was not possible to accurately establish the time of the primary event. Of the total of patients evaluated by neurosurgery, $65 \%$ needed a second evaluation due to clinical worsening, when the procedure was indicated. The number of

Table 2. Socio-demographic characteristics of the patients included in the study.

\begin{tabular}{lc}
\hline Demographic variables & $\mathrm{n}=20$ \\
\hline Age (years)* & $52.7 \pm 7.4$ \\
Sex (\%)** & $11(55)$ \\
Male & $9(45)$ \\
Female & $15(75)$ \\
Skin color (\%)** & $5(25)$ \\
White & \\
Black & \\
\hline *Continuous variable expressed as average & \pm standard deviation; \\
**Categorical variables expressed as $n(\%)$. &
\end{tabular}

CTs before surgery was 2 [1.2-3], until an eventual definitive hypodense area was established. The time interval between the last CT and the surgical procedure was 7.7 [2.3-14.8] hours, considering the time elapsed from the neurosurgical evaluation and the availability of the operating room for the performance of the operation. The median mRS 30 days after the procedure was 4 [3.3-6] for all patients undergoing DC. Those who were discharged with neurological deficits had a mRS score 4 [3-4].

The clinical and surgical characteristics are shown in Table 3.

The DC mortality rate evaluated 30 days postoperatively (Figure) was 30\% (95\%CI 14.5-51.9).

Table 3. Clinical and surgical characteristics of the patients included in the study.

\begin{tabular}{lc}
\hline Variables & $n=20$ \\
\hline Ictus time x admission (hours)* & $13.2 \pm 1.4$ \\
\hline Hospitalization NIHSS (score)* & $17.6 \pm 3.2$ \\
NCl evaluation x surgery (hours)** & $2.9[1.9-4.4]$ \\
Ictus time x surgery (hours)** & $28.1[21.2-50.6]$ \\
Clinical worsening after 1st NCl evaluation (\%)*** & $13(65)$ \\
No of CTs before surgery** & $2[1.2-3]$ \\
Time interval between surgery and last CT (hours)** & $7.7[2.3-14.8]$ \\
Rankin (30 days score)** & $4[3.3-6]$ \\
Rankin (discharge score)** & $4[3-4]$ \\
Discharge rate (\%)*** & $14(70)$ \\
Death rate (\%)*** & $6(30)$ \\
\hline Hospitalization time (days)** & $21.5[9.5-34.5]$ \\
\hline
\end{tabular}

NIHSS: National Institutes of Health Stroke Scale; $\mathrm{NCl}$ : neurosurgery; CT: computerized tomography. *Continuous variable expressed as average \pm standard deviation; ${ }^{\star *}$ Asymmetrical variables expressed as median and interquartile interval; ${ }^{* \star}$ Categorical variables expressed as $n(\%)$.

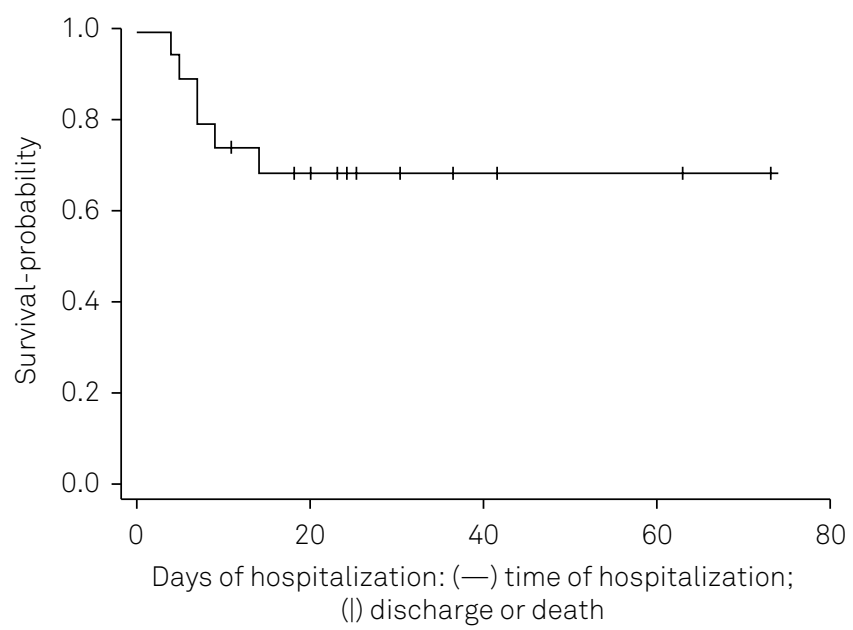

Figure. Kaplan-Meier curve of in-hospital mortality of patients included in the study. 


\section{DISCUSSION}

To the best of our knowledge, this is the first study that assessed the DC outcome in malignant MCA infarction in an academic hospital in Brazil, considered a reference for the treatment of stroke patients nationwide. As a main finding, there was a $30 \%$ mortality rate among patients subjected to DC. Another Brazilian study, involving 34 patients, showed similar data, with a surgical mortality rate of $26 \%^{11}$.

Previous studies ${ }^{12,13}$ have recommended performing DC for the treatment of malignant cerebral infarction in patients aged 60 years or less, before clinical or radiological signs of brain herniation, within a time frame of 48 hours. In the present study, we had an average age of $52.7 \pm 7.4$ years, including three patients over 60 years of age. The average age of patients in the randomized controlled trials HeADDFIRST ${ }^{14}$, DECIMAL $^{9}$, DESTINY ${ }^{8}$ and HAMLET $^{7}$ was, respectively, 54.6; 43.4; 44.6 and 48.2 years.

Several studies have reported that age should be considered an important factor in patients to be submitted to the surgical procedure ${ }^{15,16,17}$. The decision for patients over 60 years of age must be individualized: there is great controversy regarding this cut-off point, as some authors describe benefits beyond this age-group ${ }^{12,15,18,19}$, while others report unfavorable outcomes ${ }^{6,7,8,9}$. In the present study, three patients over 60 years of age were included. A Chinese study evaluated the effectiveness of DC in patients over 80 years of age, concluding that DC, even in patients in this age group, may increase the survival rate without severe compromise of functionality ${ }^{18}$. In a recent American study, involving more than 1,600 patients, the authors reported the results according to age as uni- and multivariate analysis, not taking into account this variable as a significant predictor of clinical outcome ${ }^{20}$.

In the present study, an average of 3,5 points in the mRS score was found among survivors, a value considered acceptable (mild to moderately severe disability), as those patients probably would have died without surgical treatment. A recent cross-sectional and multicenter study aimed at identifying the opinion of more than 1,800 physicians, with clinical experience in MCA treatment, about what they considered an acceptable disability. The majority of respondents (79.3\%) considered acceptable mRS scores of 3 or greater ${ }^{21}$.

Individuals affected by a malignant ischemic event are seldom discharged without severe clinical deficits; this data is in accordance with a recent study of meta-analysis carried out in China, involving more than 700 patients, that considered an mRS of 4 a good functional result ${ }^{10}$.

In two out of the 20 patients in the sample, it was not possible to clearly determine the approximate time lapse from stroke to admission in the emergency unit of the hospital. In those where this information could be obtained, the average time was $13.2 \pm 1.4$ hours. Such data has a potential clinical significance, since the longer the time between the ictus, the diagnosis and the operation procedure, the smaller the chances of positive outcomes are.

The median time between the ictus and the operation was 28.1 [21.2-50.6] hours, different from another Brazilian study with 34 cases that presented an average time of 2.05 days $^{11}$. We highlight that, among the studied patients, three were submitted to the procedure late, due to the lack of a clear definition of the MCA territory hypodensity $>2 / 3$. This was confirmed by the number of CTs before the operation (2.2 [1-5]), data that suggests the caution with which the surgical procedures were indicated. In the same manner, the time elapsed from the last CT to the surgical procedure was 8.7 [2-23] hours, somewhat large, but justified by the logistical difficulties, since this hospital is a teaching hospital with a very busy emergency department requiring surgery, and a waiting list for the available operating rooms. Even without immediate availability of an operating room, the neurosurgery team remained in constant contact, evaluating and handling of these patients, which can be underlined by the time-elapsed hours between the last neurosurgery evaluation and the surgical procedure: up to $50 \%$ of the evaluated patients were subjected to surgery within 2,9 hours.

The median time of hospitalization, including the ICU and infirmary stay, was 21.5 [9.5-34.5] days. In a retrospective study carried out in France, where ten patients subjected to DC were evaluated, the average ICU stay was 22 days $(3-58)^{22}$. It is important to highlight that the time of the present study was measured from the time of hospitalization to the final outcome, including one (5\%) who was hospitalized for an elective tracheoplasty and fifteen days later suffered a stroke.

An average NIHSS score of $17.6 \pm 3.2$ was found upon hospitalization, which demonstrates the clinical severity of the patients. In a recent Japanese study involving 355 patients, an average NIHSS score of 18 points (minimum 0 - maximum 38) was shown at admission, and in the DC group it was $21^{5}$. These results suggest that the patients in our study followed strict selection criteria for the surgical procedure.

A real limitation of this study is the type of outlining used, since the group of patients subjected to the DC procedure was not compared to any other patients subjected to conservative treatment, as reported in the major randomized clinical trials ${ }^{7,89,12}$

In conclusion, when performed based on a strict institutional protocol, DC is a great treatment option for the decrease of mortality and morbidity rates. 
1. Musabelliu E, Kato Y, Imizu S, Oda J, Sano H. Surgical treatment of patients with ischemic stroke decompressive craniectomy. In: Rodríguez JCG, editor. Acute ischemic stroke. Rijeka: InTech; 2012. p. 165-86.

2. Mellado TP, Castillo FL, Campos PM, Bugedo TG, Dougnac LA, Andresen HM. [Decompressive hemicraniectomy for malignant middle cerebral artery infarction: report of two cases]. Rev Med Chil. 2005;133(4):447-52. Spanish.

3. Neugebauer H, Heuschmann PU, Jüttler E. DEcompressive surgery for the Treatment of malignant INfarction of the middle cerebral arterY - Registry (DESTINY-R): design and protocols. BMC Neurol. 2012;12(1):1-6. https://doi.org/10.1186/1471-2377-12-115

4. Ropper AH. Hemicraniectomy: to halve or halve not. N Engl J Med. 2014;370(12):1159-60. https://doi.org/10.1056/NEJMe1315721

5. Suyama K, Horie N, Hayashi K, Nagata I. Nationwide survey of decompressive hemicraniectomy for malignant middle cerebral artery infarction in Japan. World Neurosurg. 2014;82(6):1158-63. https://doi.org/10.1016/j.wneu.2014.07.015

6. Chen CC, Cho DY, Tsai SC. Outcome of and prognostic factors for decompressive hemicraniectomy in malignant middle cerebral artery infarction. J Clin Neurosci. 2007;14(4):317-21. https://doi.org/10.1016/j.jocn.2005.05.024

7. Hofmeijer J, Kappelle LJ, Algra A, Amelink GJ, Gijn J, Worp HB et al. Surgical decompression for space-occupying cerebral infarction (the Hemicraniectomy After Middle Cerebral Artery infarction with Life-threatening Edema Trial [HAMLET]): a multicentre, open, randomised trial. Lancet Neurol. 2009;8(4):326-33. https://doi.org/10.1016/S1474-4422(09)70047-X

8. Jüttler E, Schwab S, Schmiedek P, Unterberg A, Hennerici M, Woitzik $J$ et al. Decompressive surgery for the treatment of malignant infarction of the middle cerebral artery (DESTINY): a randomized, controlled trial. Stroke. 2007;38(9):2518-25. https://doi.org/10.1161/STROKEAHA.107.485649

9. Vahedi K, Vicaut E, Mateo J, Kurtz A, Orabi M, Guichard JP et al. Sequential-design, multicenter, randomized, controlled trial of early decompressive craniectomy in malignant middle cerebral artery infarction (DECIMAL Trial). Stroke. 2007;38(9):2506-17. https://doi.org/10.1161/STROKEAHA.107.485235

10. LuX, Huang B, Zheng J, Tao Y, Yu W, Tang L et al. Decompressive craniectomy for the treatment of malignant infarction of the middle cerebral artery. Sci Rep. 2014;4(7070):7070. https://doi.org/10.1038/srep07070

11. Nobre MC, Monteiro M, Albuquerque AC, Veloso AT, Mendes VA, Silveira MF et al. [Decompressive craniectomy for treatment of intracranial hypertension secondary to large ischemic cerebral infarction: analysis of 34 cases]. Arq Neuropsiquiatr. 2007;65(1):107-13. Portuguese. https://doi.org/10.1590/S0004-282X2007000100022
12. Jüttler E, Unterberg A, WoitzikJ, Bösel J, Amiri H, Sakowitz OW et al. Hemicraniectomy in older patients with extensive middle-cerebral artery stroke. N Engl J Med. 2014;370(12):1091-100. https://doi.org/10.1056/NEJMoa1311367

13. Huttner HB, Schwab S. Malignant middle cerebral artery infarction: clinical characteristics, treatment strategies, and future perspectives. Lancet Neurol. 2009;8(10):949-58. https://doi.org/10.1016/S1474-4422(09)70224-8

14. Frank JI, Schumm LP, Wroblewski K, Chyatte D, Rosengart AJ, Kordeck C et al. Hemicraniectomy and durotomy upon deterioration from infarction-related swelling trial: randomized pilot clinical trial. Stroke. 2014;45(3):781-7. https://doi.org/10.1161/STROKEAHA.113.003200

15. Fiorot Junior JA, Silva GS, Cavalheiro S, Massaro AR. Use of decompressive craniectomy in the treatment of hemispheric infarction. Arq. Neuropsiquiatr. 2008;66(2A):204-8. https://doi.org/10.1590/S0004-282X2008000200012

16. Gupta R, Connolly ES, Mayer S, Elkind MS. Hemicraniectomy for massive middle cerebral artery territory infarction: a systematic review. Stroke. 2004;35(2):539-43. https://doi.org/10.1161/01.STR.0000109772.64650.18

17. Antuña-Ramos A, Alvarez-Vega MA, Seijo-Fernández F, Calleja-Puerta S, González-Delgado M, Torres-Campa JM et al. [Surgical treatment of the stroke in the middle cerebral artery]. Rev Neurol. 2009;49(7):354-8. Spanish.

18. Zhao J, Su YY, Zhang Y, Zhang YZ, Zhao R, Wang L et al. Decompressive hemicraniectomy in malignant middle cerebral artery infarct: a randomized controlled trial enrolling patients up to 80 years old. Neurocrit Care. 2012;17(2):161-71. https://doi.org/10.1007/s12028-012-9703-3

19. Inamasu J, Kaito T, Watabe T, Ganaha T, Yamada Y, Tanaka T et al. Decompressive hemicraniectomy for malignant hemispheric stroke in the elderly: comparison of outcomes between individuals 61-70 and $>70$ years of age. J Stroke Cerebrovasc Dis. 2013;22(8):1350-4. https://doi.org/10.1016/j.jstrokecerebrovasdis.2013.02.008

20. Daou B, Kent AP, Montano M, Chalouhi N, Starke RM, Tjoumakaris $S$ et al. Decompressive hemicraniectomy: predictors of functional outcome in patients with ischemic stroke. J Neurosurg. 2016;124(6):1773-9. https://doi.org/10.3171/2015.6.JNS15729

21. Neugebauer H, Creutzfeldt CJ, Hemphill JC 3rd, Heuschmann PU, Jüttler E. DESTINY-S: attitudes of physicians toward disability and treatment in malignant MCA infarction. Neurocrit Care. 2014:21(1):27-34. https://doi.org/10.1007/s12028-014-9956-0

22. Vahedi K, Benoist L, Kurtz A, Mateo J, Blanquet A, Rossignol M et al. Quality of life after decompressive craniectomy for malignant middle cerebral artery infarction. J Neurol Neurosurg Psychiatry. 2005;76(8):1181-2. https://doi.org/10.1136/jnnp.2004.058537 